\title{
Acute Promyelocytic Leukemia in a Pregnant
}

\author{
Trelles R, Bobes A*, Anguita E, Garcia-Roa M, \\ Mateo $M$ and Mart nez $R$ \\ Department of Hematology, Clinico San Carlos Hospital \\ Madrid, Spain \\ *Corresponding author: Alejandro Bobes, \\ Department of Hematology, Clinico San Carlos Hospital \\ Madrid, Spain
}

Received: January 24, 2017; Accepted: February 25, 2017; Published: February 28, 2017

\begin{abstract}
Acute promyelocytic leukemia (APL) is a subtype of acute myeloid leukemia characterized by a life-threatening coagulopathy that becomes a real emergency. However, it is also the first malignant disease highly curable with targeted therapy (all-trans retinoic acid, or ATRA) directed against a unique genetic abnormality, $\mathrm{t}(15 ; 17) / P L M-R A R \alpha$, with the potential to correct the coagulation disorder. As a consequence, as soon as the diagnosis is suspected, it is necessary to start ATRA treatment without delay. Nevertheless, ATRA is a known teratogenic drug, therefore pregnancy in this disease is a challenge. We report the case of a pregnancy woman with APL. The decision between all the specialists is complicated. Pregnancy women treated in her last trimester of pregnancy could achieve a good outcome with ATRA, not only for the woman but also for the baby.
\end{abstract}

Keywords: Acute promyelocytic leukemia; Pregnancy; All-trans retinoic acid; Teratogenic

\section{Case Presentation}

We report the case of a 41-year-old woman diagnosed of APL at 35 weeks' gestation after several days of gingivorragia, epistaxis and vascular purpura. A blood test revealed a severe thrombocytopenia $(3.6 \times 10 \mathrm{E} 3 / \mu \mathrm{L})$, anemia (hemoglobin $8.9 \mathrm{~g} / \mathrm{dl})$ and leukopenia $(3.5 \times 10$ $\mathrm{E} 3 / \mu \mathrm{L})$. Coagulation test was in the normal range, except from a low fibrinogen of $140 \mathrm{mg} / \mathrm{dl}$. Biochemical analysis including creatinine was normal [1-3].

Bone marrow aspiration showed $95 \%$ of abnormal promyelocytes of total cellularity. FISH study demonstrated a $\mathrm{t}(15 ; 17)$ and RT-PCR was positive for $P M L / R A R \alpha$ BCR2.

Gynecological examination was consistent with a healthy fetus.

She started therapy with ATRA $45 \mathrm{mg} / \mathrm{m}^{2}$ and $12 \mathrm{mg} / \mathrm{m}^{2}$ idarubicin (AIDA protocol) associated with supportive measures including blood and platelet transfusions and intravenous fibrinogen administration.

Once the coagulopathy was solved, 9 days after begining chemotherapy, she had an accomplished cesarean birth. The newborn was totally healthy with a weight of $2700 \mathrm{~g}$ and with a mild pancytopenia. After surgery, an antibiotic prophylaxis treatment was initiated following the hospital prophylaxis antibiotic guidelines. After several days and in the context of febrile neutropenia, the surgical wound became infected. Several days after beginning antibiotic treatment and after recovering blood counts, she was discharged in complete remission revealed by bone marrow aspiration and molecular biology.

Then, she started consolidation therapy with Mitoxantrone, ATRA and Idarubicine according to the previously mentioned protocol with no complications except from febrile neutropenia.

She continued with maintenance treatment with ATRA, Mercaptopurine and Metotrexate for 9 cycles. She ended up treatment in January 2008 with no relapse till today (102 months).

\section{Discussion}

APL is characterized by a good response to therapy with teratogenic ATRA and chemotherapy once the potential mortal coagulopathy is under control. Therefore, treatment of APL during pregnancy is not easy. ATRA is not recommended at the first trimester of pregnancy for the proper development of the fetus, but treatment during second or third trimester are supposedly safe [4-6]. Additionally, chemotherapy is reasonably safe in the last period of pregnancy [6]. APL's coagulopathy adds a high level of complexity. The election of the best moment for delivery should consider the resolution of it with the lowest drug related effects and we considered cesarean as the best method to select the moment in a precise clinically controlled environment.

\section{Conclusion}

Treatment cannot be delayed in APL. This includes teratogenic drugs. Therefore, a therapeutic abortion would be recommended in the first trimester of pregnancy. However, during the second and third trimester ATRA plus chemotherapy associated with supportive care and a close surveillance of the mother and the fetus may finish in a good outcome. Our case and the experience reflected in the literature suggest that an accurate control of the treatment required to solve the coagulopathy prevents ATRA syndrome and produces minimal drug related effects for the mother and fetus. We believe that cesarean should be considered to control the most suitable moment of delivery and to prevent and treat possible complications that may arise.

\section{References}

1. Carradice D, Austin N, Bayston K, Ganly PS. Successful treatment of acute promyelocytic leukaemia during pregnancy. Clin Lab Haematol. 2002; 24: 307- 311.

2. Giagounidis AA, Beckmann MW, Giagounidis AS, Aivado M, Emde T Germing $U$, et al. Acute promyelocytic leukemia and pregnancy. Eur $\mathrm{J}$ Haematol. 2000. 64: 267-271.

3. Chelghoum Y, Vey N, Raffoux E, Huguet F, Pigneux A, Witz B, et al. Acute leukemia during pregnancy: a report on 37 patients and a review of literature. Cancer. 2005; 104: 110-117. 
4. Esteve J, Escoda L, Martín G, Rubio V, Díaz-Mediavilla J, González M, et al. Spanish Cooperative Group PETHEMA. Outcome of patients with acute promyelocytic leukemia failing to front-line treatment with all-trans retinoic acid and anthracycline-based chemotherapy (PETHEMA protocols LPA96 and LPA99): benefit of an early intervention. Leukemia. 2007; 21: 446-452.
5. Yang D, Hladnik L. Treatment of acute promyelocytic leukemia during pregnancy. Pharmacotherapy. 2009; 29: 709-724.

6. Milojkovic D, Apperley JF. How I treat leukemia during pregnancy. Blood 2014; 123: 974-984.
Ann Hematol Oncol - Volume 4 Issue 3 - 2017

ISSN : 2375-7965 | www.austinpublishinggroup.com

Bobes et al. (C) All rights are reserved
Citation: Trelles R, Bobes A, Anguita E, Garcia-Roa M, Mateo M and Mart nez R. Acute Promyelocytic Leukemia in a Pregnant. Ann Hematol Oncol. 2017; 4(3): 1138. 